\title{
Changes in the Neuronal Control of the Urinary Bladder in a Model of Radiation Cystitis
}

\author{
Daniel Giglio, Lucie Podmolíková, and Gunnar Tobin \\ Department of Pharmacology, Institution of Neuroscience and Physiology (D.G., L.P., G.T.) and Department of Oncology, \\ Institution of Clinical Sciences, University of Gothenburg, Gothenburg, Sweden (D.G.); and Department of Medical Biochemistry, \\ Faculty of Medicine, Charles University, Hradec Králové, Czech Republic (L.P.)
}

Received November 17, 2017; accepted February 20, 2018

\begin{abstract}
Currently, we have assessed the neuronal control of the urinary bladder in radiation cystitis and whether interstitial cells contribute to the condition. Fourteen days after bladder irradiation (20 Gy), rats were sedated and the urinary bladder was cut into two sagittal halves where electrical field stimulation (EFS; $5-20 \mathrm{~Hz}$ ) was applied on the pelvic nerve afferents or stretch $(80 \mathrm{mN})$ on one-half of the bladder, while contractions were registered on the contralateral half of the bladder in the absence and presence of increasing doses of imatinib (1-10 mg/kg; inhibitor of c-kit-positive interstitial cells), atropine ( $1 \mathrm{mg} / \mathrm{kg}$; to block muscarinic $\mathrm{M}_{3}$ receptors), or pyridoxalphosphate-6-azophenyl-2',4'-disulfonic acid (2 mg/kg; P2 21 purinoceptor antagonist). Urinary bladders were also excised for organ bath experiments, Western blot, quantitative polymerase
\end{abstract}

chain reaction, and immunohistochemistry. In vivo, EFS contractions were enhanced after irradiation, and imatinib $(1-10 \mathrm{mg} / \mathrm{mg})$ inhibited contractions by EFS and stretched dose-dependently in controls but not in irradiated bladders. In the irradiated bladder in vitro, atropine resistance was increased and imatinib (100 $\mu \mathrm{M})$ inhibited contractions by EFS and agonists (ATP, methacholine) in irradiated bladders and controls. The urinary bladder expressions of $\mathrm{P} 2{ }_{x 1}$ purinoceptors, muscarinic $\mathrm{M}_{3}$ receptor, choline acetyltransferase, c-kit, and the agonist of c-kit, stem cell factor, were not changed by irradiation. In conclusion, bladder irradiation affects several levels of neuronal control of the urinary bladder. Interstitial cells may contribute to some of the symptoms associated with radiation cystitis.

\section{Introduction}

Radiation cystitis is a common and troublesome side effect of radiation toward tumors in the pelvic region. The condition is characterized by bladder urgency and frequency, bladder pain, and bleeding (Zwaans et al., 2016). In most cases, radiation cystitis is transient, but chronic cystitis may also develop, with severe impact on quality of life (Zwaans et al., 2016). The underlying mechanisms of how chronic radiation cystitis develops are not understood. In animal models of radiation cystitis, changes in cholinergic and purinergic contractile effects of the detrusor have been demonstrated (Michailov et al., 1991; Vale et al., 1994). In addition to changes in neuronal signaling, structural changes may also contribute to bladder disturbances (e.g., the amount of collagen is increased in the bladder wall after irradiation) (Soler et al., 2011). In a rat model for radiation cystitis, we showed that bladder irradiation induces long-lasting changes

The present study was sponsored by the King Gustav V Jubilee Clinic Cancer Research Foundation, Göteborg, Sweden [Grant number 2016:79] and the Magnus Bergvall Foundation.

https://doi.org/10.1124/jpet.117.246371. in oxidative stress and cytokine levels in the urinary bladder (Giglio et al., 2016; Oscarsson et al., 2017).

The regulation of the urinary bladder control is complex, involving the autonomic and somatic nervous systems. Studies have shown the existence of interstitial cells in the bladder, which may regulate afferent neuronal control in the urinary bladder in a manner similar to that in interstitial cells of Cajal (pacemaker cells) in the gastrointestinal tract (Kubota et al., 2006). The cells are located in the lamina propria and between smooth muscles bundles, and seem to interact with smooth muscle contraction and with sensory neurons and urothelium (Brading and McCloskey, 2005). The interstitial cells are characterized by their expression of c-kit (McCloskey and Gurney, 2002; van der AA et al., 2004). However, interstitial cells seem to belong to different subpopulations of cells where some cells express c-kit, whereas others do not (Gevaert et al., 2014). The c-kit inhibitor imatinib may antagonize urinary bladder contractions induced by electrical field stimulation (EFS) of the pelvic nerve in vivo but not those induced by acetylcholine in vitro (Min et al., 2011). Changes in interstitial cells have been suggested to contribute to the pathogenesis of diabetic bladder dysfunction, overactive bladder, and bladder dysfunction after spinal cord injury (Kim et al., 2011; Deng et al., 2013; Canda et al., 2014).

ABBREVIATIONS: $\alpha, \beta$-meATP, $\alpha, \beta$-methylene ATP; ANOVA, analysis of variance; ChAT, choline acetyltransferase; DAB, $3^{\prime}$-diaminobenzidine; DAPI, 4',6-diamidino-2-phenylindole; EFS, electrical field stimulation; HRP, horseradish peroxidase; ID $\mathrm{D}_{50}$, inhibitory dose 50; IHC, immunohistochemistry; PDGFr $\beta$, platelet-derived growth factor receptor $\beta$; PPADS, pyridoxalphosphate-6-azophenyl-2', $4^{\prime}$-disulfonic acid; qPCR, quantitative polymerase chain reaction; SCBT, Santa Cruz Biotechnology; SCF, stem cell factor; TBST, Tris-buffered saline containing 0.3\% Tween 20; WB, Western blot. 
In our animal model for radiation cystitis, we observed an enhanced micturition frequency and smaller voiding volumes in animals exposed to bladder radiation (Giglio et al., 2016). In the present study, we assessed the neurologic control of the urinary bladder in the state of radiation cystitis in a unique in vivo model and in vitro. Our results show that bladder irradiation leads to significant changes in the functional control of the urinary bladder.

\section{Material and Methods}

Bladder Irradiation. The study was approved by the Animal Ethics Committee of the University of Gothenburg. Adult female rats (250-300 g; CD IGS rat; Charles River, Freiburg, Germany) were housed in a temperature-controlled facility with a day and night cycle and had food and water available ad libitum. The rats were anesthetized with pentobarbitone $(50 \mathrm{mg} / \mathrm{kg}$, i.m.) and medetomidine $(10 \mu \mathrm{g} / \mathrm{kg}$, i.p.), and then were placed in the supine position and the urinary bladder was then exposed to one fraction of 20 Gy using a linear accelerator with 6-mV photon energy (Varian Medical Systems Inc., Palo Alto, CA). To avoid exposure of the spinal cord, the radiation was administered using two side fields. Control rats were only sedated. No significant change in rat body weight was observed 2 weeks after bladder irradiation compared with controls (315 \pm 11 and $333 \pm 15 \mathrm{~g}$, respectively; $n=11-14$ ).

In Vivo Studies of the Anesthetized Rat. On the day of the experiment, the rat was sedated with isoflurane and a small sagittal incision was made on the lower abdomen of the rat over the urinary bladder. The experiment was conducted as previously described with an in situ half bladder preparation (Aronsson et al., 2014, 2015). In brief, the urinary bladder was cut into two sagittal parts (A and B). A small ligature was tied to the apical part of sagittal part $A$, and the ligature was connected to an adjustable isometric force transducer (Linton Instrumentation, Norfolk, UK). A ligature was tied to the contralateral apical part of sagittal part B, and during the experiment for force application weights were tied to the loose end of the ligature. Both parts were fixated at the urethral end to the abdominal wall. The pelvic nerve innervating sagittal part B was exposed at surgery, and an electrode was placed onto the nerve. A basal tension of 8-15 mN was applied on sagittal part A. The anesthesia was successfully reduced until afferent responses occurred in response to $20-\mathrm{Hz}$ afferent stimulation but was still keeping the animal anesthetized. Intravenous drug administrations were administered via a cannula placed in the femoral vein. At the start of the experiment, methacholine $(5 \mu \mathrm{g} / \mathrm{kg})$ was administered intravenously. This was followed by connecting an $80 \mathrm{mN}$ weight to sagittal part $\mathrm{B}$ for 2 minutes, and contractions of the sagittal part A were then registered. The afferent pelvic nerve to sagittal part B was then stimulated with EFS at 5, 10, and $20 \mathrm{~Hz}$ (2 minutes per frequency) at supramaximal voltage, and contractions of sagittal part A were registered. The protocol was then repeated in the presence of increasing administered doses of the c-kit inhibitor imatinib $(1,3$, and $10 \mathrm{mg} / \mathrm{kg})$. In an additional setup of experiments, force $(20 \mathrm{~Hz})$ and EFS $(20 \mathrm{~Hz})$ were applied as described above in the absence and presence of administered doses of imatinib $(10 \mathrm{mg} / \mathrm{kg})$, the nonselective muscarinic receptor antagonist atropine (1 mg/kg), and the P2 purinoceptor antagonist pyridoxalphosphate-6azophenyl-2',4'-disulphonic acid (PPADS; $2 \mathrm{mg} / \mathrm{kg}$ ). All antagonists were equilibrated for 3 minutes before tension and EFS were given. Control experiments of the in vivo model showed no change over time in EFS- or methacholine-induced bladder responses.

In Vitro Organ Bath Studies. Rats were anesthetized with pentobarbitone, following which urinary bladders were removed. The rats were subsequently euthanized by an overdose of pentobarbitone. Full-thickness urinary bladder strips $(6 \times 2 \mathrm{~mm})$ were excised from the trigonal area of the urinary bladder. The bladder strip was then mounted between two steel rods, one of which was adjustable and connected to an isometric force transducer (Linton Instrumentation), in 25-ml organ baths. The organ baths contained Krebs solution with the following composition (millimolar): $\mathrm{NaCl} 118, \mathrm{KCl} 4.6, \mathrm{CaCl} 21.25$, $\mathrm{KH} 2 \mathrm{PO} 4$ 1.15, MgSO4 1.15, NaHCO3 25, and glucose 5.5, which was gassed with $5 \% \mathrm{CO}_{2}$ in $\mathrm{O}_{2}$ at $\mathrm{pH} 7.4$ and heated by a thermoregulated water circuit $\left(37^{\circ} \mathrm{C}\right)$. The bladder strip was then tensioned so that a stable tension of about $5 \mathrm{mN}$ was achieved after 60 minutes of equilibrium. During the equilibrium, the organ baths were washed every 15 minutes. A high- $\mathrm{K}^{+}$solution (containing $124 \mathrm{mM} \mathrm{K}$ obtained by exchanging $\mathrm{Na}^{+}$for equimolar amounts of $\mathrm{K}^{+}$) was administered in the beginning of each experiment for a reference contraction value and to assess the viability of each individual bladder strip. All drugs were dissolved in distilled $\mathrm{H}_{2} \mathrm{O}$ and added at a volume of $125 \mu \mathrm{l}$ to the organ baths. EFS was applied at varying frequencies (between 0.2 and $40 \mathrm{~Hz}$ ) at supramaximal voltage $(50 \mathrm{~V})$ delivered as square wave pulses with a duration of $0.5 \mathrm{~ms}$ continuously until the peak response was obtained (stimulator: STM100C; Linton Instrumentation). To desensitize P2 2 purinoceptors, $\alpha, \beta$-meATP $(10 \mu \mathrm{M})$ was administered repeatedly every 10 minutes until no contractile response was attained with the agonist, which occurred after three administrations. Control organ bath experiments showed no change over time in EFS- or methacholine-induced bladder responses. Data were recorded using an MP100WSW Data Acquisition System and Acquire software (BIOPAC Systems, Inc., Goleta, CA).

Immunohistochemistry. Bladder sections of $6 \mu \mathrm{m}$ were deparaffined in xylene and hydrated in decreasing concentrations of ethanol $(99 \%-95 \%)$ followed by washing the sections in tap water. Antigen retrieval was performed by boiling the sections in citrate buffer $(\mathrm{pH}$ 7.5). Sections were then incubated with $\mathrm{CuSO}_{4}$ solution (SigmaAldrich, St. Louis, MO) for 2 hours to block autofluorescence followed by blocking unspecific binding with $5 \%$ goat (or donkey when the antibody was raised in goat) serum for 1 hour. Sections were incubated overnight at $4^{\circ} \mathrm{C}$ with the primary antibody diluted in $1 \%$ goat (or donkey) serum in phosphate-buffered saline. On the next day, sections were incubated with the secondary antibody [1:250 diluted in 1\% goat serum (or donkey) in phosphate-buffered saline] for 1 hour. Sections were then dehydrated in ethanol (95\%-99\%), and cover glasses were mounted on the sections with ProLong Gold Antifade Reagent with DAPI (4',6-diamidino-2-phenylindole; Life Technologies Ltd, Paisley, UK). Horseradish peroxidase (HRP)/3'-diaminobenzidine (DAB) immunohistochemistry (IHC) was performed according to the instructions of the manufacturer [Mouse and Rabbit Specific HRP/DAB (ABC) Detection IHC Kit, cat. no. ab64264; Abcam, Cambridge, UK]. Sections were deparaffined, hydrated, and blocked with hydrogen peroxide. Antigen retrieval was then performed as described above. Protein block was applied on the sections, which was followed by incubating the sections overnight with the primary antibody. The next day, sections were incubated with biotinylated goat anti-polyvalent followed by streptavidin peroxidase. DAB chromogen mixed with DAB substrate was then applied on the sections for 5 minutes. Sections were counterstained with Mayer's Hematoxylin Solution (Histolab Products AB, Göteborg, Sweden), washed in tap water, and dehydrated in increasing concentrations of ethanol and xylene. Sections were then covered with Pertex Mounting Medium (Histolab Products $\mathrm{AB}$ ) and coverslips.

Western Blot. Bladder specimens were homogenized in homogenization buffer and centrifuged, and the supernatants were collected. Protein concentrations of the samples were quantified with the Pierce BCA Protein Assay Kit (Thermo Fisher Scientific, Rockford, IL). After adding NuPAGE LDS Sample Buffer (25\% of total volume; Life Technologies, Carlsbad, CA), NuPAGE Reducing Agent ( $10 \%$ of total volume; Life Technologies), and distilled $\mathrm{H}_{2} \mathrm{O}$ (to equalize protein concentrations between samples), the samples were heated to $70^{\circ} \mathrm{C}$ for 10 minutes. Samples were loaded on a NuPAGE 4\%-12\% Bis-Tris gel (Life Technologies) followed by electrophoresis at $200 \mathrm{~V}$ for 60 minutes under reducing conditions in $3-(N$ morpholino)propanesulfonic acid (MOPS) buffer (Life Technologies). Proteins were transferred onto a nitrocellulose membrane (Life Technologies) for 60 minutes at $30 \mathrm{~V}$. The membranes were thereafter 
washed in Tris-buffered saline containing $0.3 \%$ Tween 20 (TBST; Sigma-Aldrich) and blocked for 60 minutes with TBST containing 5\% nonfat milk. The membranes were incubated overnight with the primary antibody (diluted in 3\% goat serum in TBST; see list below). The following morning after washes in TBST, the membranes were incubated with the secondary antibody (diluted in 5\% nonfat milk in TBST; see list below) for 1 hour. The membranes were washed in TBST, and the binding of the antibody was then detected with the Amersham ECL Plus Western Blotting System (GE Healthcare, Little Chalfont, UK), visualized by using the Fujifilm (Stockholm, Sweden) Image Reader LAS-1000 Pro version 2.6. Membranes were stripped in Restore Western Blot Stripping Buffer (Thermo Fisher Scientific) and incubated with other primary antibodies followed by the protocol described above.

The following antibodies were used: Alexa Fluor 488 donkey anti-goat IgG $(\mathrm{H}+\mathrm{L}) * 2 \mathrm{mg} / \mathrm{ml}$ (IHC 1:250; cat. no. A11005; Life Technologies); Alexa Fluor 488 goat anti-rabbit IgG $(\mathrm{H}+\mathrm{L}) * 2 \mathrm{mg} / \mathrm{ml}$ (IHC 1:250; cat. no. A11008; Life Technologies); goat anti-CD34 [IHC 1:100; cat. no. sc-7045; Santa Cruz Biotechnology (SCBT), Santa Cruz, CA]; goat anti-CD39L1 (IHC 1:100; cat. no. sc-160222; SCBT); goat anti-mouse IgG (H+L)-HRP [Western blot (WB) 1:4000; cat. no. 62-6520; Life Technologies]; goat anti-rabbit IgG-HRP (WB 1:4000; cat. no. 65-6120; Life Technologies); mouse anti-c-kit (IHC 1:1000/ WB 1:2000 to 1:50; cat. no. sc365504; SCBT); rabbit anti-choactase (IHC 1:100; cat. no. sc20672; SCBT); rabbit anti-choactase (WB 1: 200; cat. no. ab178850; Abcam); rabbit anti-c-kit (IHC 1:2000-1:200/ WB 1:500; cat. no. PA5-16770; Thermo Fisher Scientific, Wilmington, DE); rabbit anti-c-kit (IHC 1:500-1:200; cat. no. sab4501648; Sigma-Aldrich); rabbit anti-muscarinic $\mathrm{M}_{3}$ receptor (IHC 1:1000/WB 1:1000; cat. no. AB9018; EMD Millipore, Billerica, MA); rabbit antiP2 1 (IHC 1:1000/WB 1:1000; cat. no. P7857; Sigma-Aldrich); rabbit anti-stem cell factor (SCF) (IHC 1:5000/WB 1:5000; cat. no. ab64677; Abcam); rabbit anti-transient receptor potential cation channel subfamily V member 1 (cat. no. ab74855; Abcam), and Texas Red goat anti-mouse IgG (H+L) (IHC 1:250; cat. no. T862; Life Technologies).

Quantitative Polymerase Chain Reaction. RNA extraction and bladder tissue homogenization were performed according to the protocol of Qiagen RNeasy (Qiagen Ltd., Manchester, UK). The quality of the RNA was assessed by running the samples on the TapeStation 2200 (Agilent Technologies, Santa Clara, CA). The DeNovix DS-11 spectrophotometer was used to assess the concentration and purity of RNA. cDNA was synthetized with the SuperScript VILO cDNA Synthesis Kit (Life Technologies), and cDNA samples were analyzed using the TaqMan gene expression assays. The quantitative polymerase chain reaction (qPCR) was performed using TATAA TaqMan Gene Expression Master Mix (Life Technologies). No template control for the qPCR was included. The qPCR was performed on the QuantStudio $12 \mathrm{~K}$ Flex (Life Technologies, Waltham, MA). The reaction mix consisted of TaqMan Gene Expression Master Mix $(5 \mu \mathrm{l} ; 2 \times)$, nuclease-free water $(2.5 \mu \mathrm{l})$, TaqMan assay $(0.5 \mu \mathrm{l} ; 20 \times)$, and template $(2 \mu \mathrm{l})$. The Uracil N-Glycosylase protocol was performed for 2 minutes $\left(95^{\circ} \mathrm{C}\right)$, activation for 10 minutes $\left(95^{\circ} \mathrm{C}\right)$, denaturation for 15 seconds $\left(95^{\circ} \mathrm{C}\right)$, and annealing and elongation for 1 minute and $40-45$ seconds $\left(60^{\circ} \mathrm{C} ; 40-45\right.$ cycles). c-kit (Rn00573942_m1; Thermo Fisher Scientific) and SCF (Rn01467260_m1; Thermo Fisher Scientific) primers were used.

Statistics. Statistical significance for paired or unpaired data was determined with the Student's $t$ test. When comparisons were made with three or more groups, one-way analysis of variance (ANOVA) or one-way repeated-measures ANOVA was performed followed by the Sidak's multiple-comparisons test. When three doses of imatinib were administered, $\mathrm{ID}_{50}$ values were calculated. $P$ values of 0.05 or less were considered to be statistically significant. Data are presented as the mean \pm S.E.M. Graphs were generated and parameters computed using the GraphPad Prism program (GraphPad Software, Inc., San Diego, CA).

\section{Results}

In Vitro Contraction Studies. Contractions in response to methacholine ( $0.1 \mathrm{nM}$ to $0.5 \mathrm{mM})$ and $\alpha, \beta$-meATP $(10 \mu \mathrm{M})$ were smaller in irradiated bladders compared with controls, whereas contractions in response to $\mathrm{KCl}(124 \mathrm{mM})$ were similar in magnitude in irradiated bladder strips and controls (Fig. 1, a-c). EFS-evoked contractions were significantly reduced in irradiated bladder strips compared with controls (Fig. 1d). EFS-evoked contractions were inhibited by atropine (10 $\mu \mathrm{M})$ and further reduced after $\alpha, \beta$-meATP $(10 \mu \mathrm{M})$ desensitization in controls (Fig. 1e). Irradiated bladder strips were notably atropine resistant, i.e., atropine $(10 \mu \mathrm{M})$ only reduced contractions at $40 \mathrm{~Hz}(P<0.01 ; n=9)$ (Fig. 1f). Imatinib $(100 \mu \mathrm{M})$ inhibited methacholine-evoked bladder contractions in controls and tended to inhibit methacholineevoked bladder contractions in irradiated bladders (Fig. 2, a and d). Imatinib $(100 \mu \mathrm{M})$ potently inhibited ATP-evoked bladder contractions $(0.3-1 \mathrm{mM})$ in irradiated bladders $(P<$ $0.01 ; n=6)$ (Fig. 2e) and tended to do so in controls $(n=8)$ (Fig. 2b). Imatinib (1-100 $\mu \mathrm{M})$ significantly inhibited EFS-evoked contractions, particularly at low frequencies $(<10 \mathrm{~Hz})$ in both irradiated bladders and controls $(n=9)$ (Fig. 2, $\mathrm{c}$ and f). The combination of imatinib $(1-100 \mu \mathrm{M})$ and atropine $(10 \mu \mathrm{M})$ blocked the predominant part of the EFS-evoked contractions in both irradiated bladders and controls ( $n=9$ ) (Fig. 2, c and f).

In Vivo Studies. Methacholine ( $5 \mu \mathrm{g} / \mathrm{kg}$ ) induced urinary bladder contractions in irradiated animals and controls of similar magnitudes $(n=10-14)$ (Fig. 3c). Stimulation of the pelvic afferent nerve with EFS $(5-20 \mathrm{~Hz})$ induced contralateral bladder contractions that were significantly stronger in irradiated animals than controls (Fig. 3, a-b). EFS at $10 \mathrm{~Hz}$ induced contractions of $14.9 \pm 6.0$ and $38.8 \pm 7.6 \mathrm{mN} / \mathrm{kg}$ in controls and irradiated rats, respectively $(P<0.05 ; n=6-10)$ (Fig. 3d). In controls, high-frequency $(20 \mathrm{~Hz})$ afferent EFSinduced contractions were antagonized by imatinib (1-10 mg/ $\mathrm{kg})$ dose dependently $\left(\mathrm{ID}_{50}=1.92\right)$, but not at lower frequencies $(5-10 \mathrm{~Hz} ; n=6$ ) (Fig. 3a). In irradiated animals, imatinib $(1-10 \mathrm{mg} / \mathrm{kg})$ did not affect afferent EFS-evoked $(5-20 \mathrm{~Hz})$ bladder contractions $(n=5)$ (Fig. 3b). The remaining part of EFS-evoked bladder contractions at $20 \mathrm{~Hz}$ in the presence of imatinib $(10 \mathrm{mg} / \mathrm{kg})$ was attenuated by atropine $(1 \mathrm{mg} / \mathrm{kg})$ and PPADS ( $2 \mathrm{mg} / \mathrm{kg})$ similarly in controls and irradiated animals; however, significance was not attained (Fig. 3, e and f). The magnitudes of stretch-evoked bladder contractions at $80 \mathrm{mN}$ were similar in irradiated animals and controls (Fig. 3, g and h). Imatinib (1-10 mg/kg) antagonized dose dependently the stretch-evoked contractions in controls $\left(\mathrm{ID}_{50}=0.75\right)$, but not in irradiated animals $(n=6)$ (Fig. 3 , g and h).

WB, q-PCR, and IHC Analyses. Since bladder irradiation induced changes in the regulation of the urinary bladder contraction, we wanted to explore whether these changes depended on changes in the expression of muscarinic $\mathrm{M}_{3}$ receptors and $\mathrm{P} 2_{\mathrm{X} 1}$ purinoceptors that mediate the predominant part of the bladder contraction. No expressional differences were, however, observed between irradiated bladders and controls (Fig. 4). Since atropine resistance was increased in irradiated bladders, we also assessed whether the expression of choline acetyltransferase (ChAT) was affected. No expressional difference of ChAT was, however, observed between irradiated bladders and controls (Fig. 4). c-kit immunostaining of bladder preparations was assessed with 

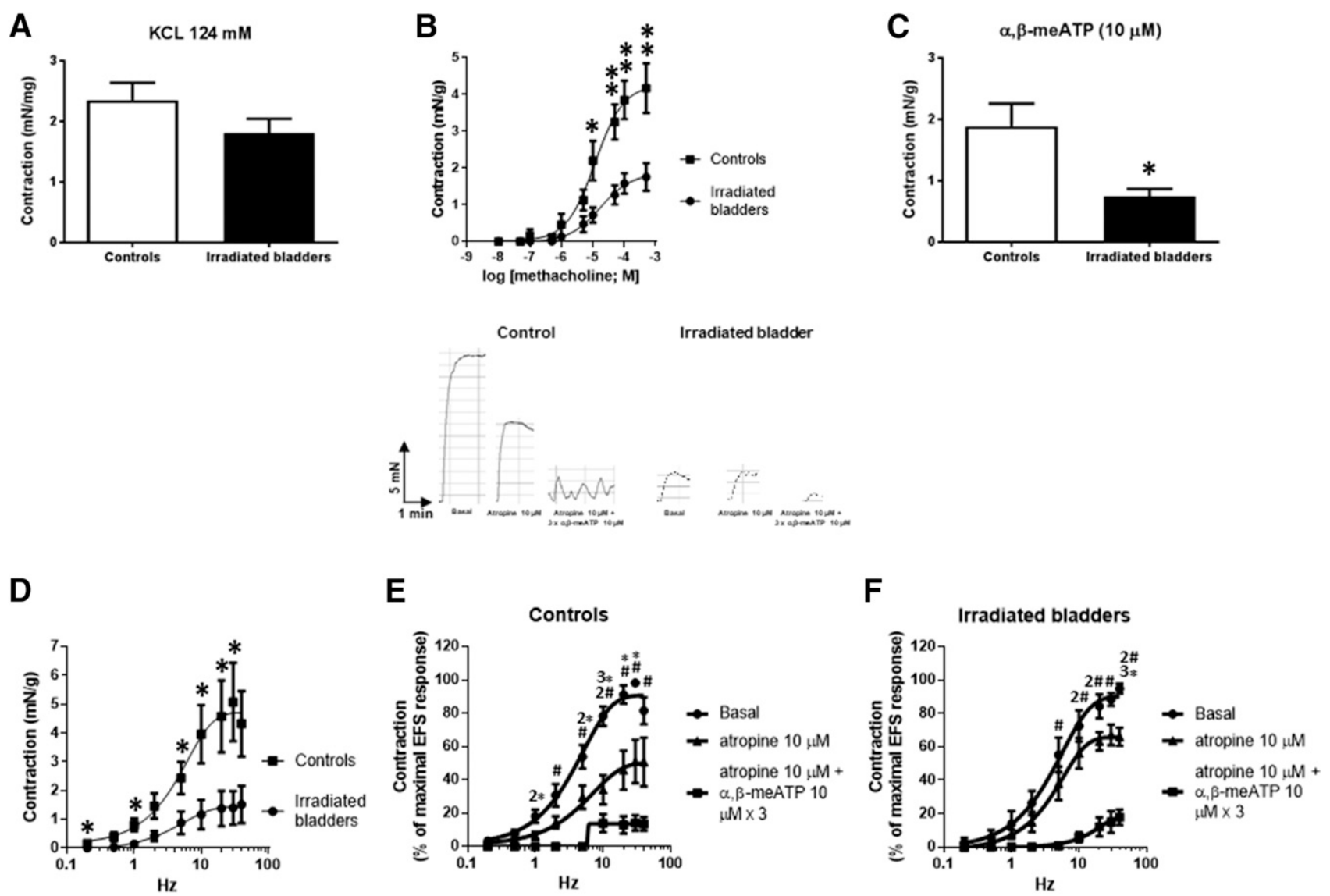

Fig. 1. Urinary bladder contractions evoked by $\mathrm{KCl}(124 \mathrm{mM} ; n=20-28)$ (a), methacholine (10 $\mathrm{nM}$ to $500 \mu \mathrm{M} ; n=7)(\mathrm{b})$, and $\alpha, \beta$-meATP (10 $\mu \mathrm{M} ; n=7-8)$ (c) in irradiated bladder preparations and controls. Urinary bladder contractions evoked by EFS $(0.2-40 \mathrm{~Hz})$ in irradiated bladders and controls $(n=6)$ (d), urinary bladder contractions evoked by EFS $(0.2-40 \mathrm{~Hz})$ in controls $(n=6)(\mathrm{e})$, and irradiated bladder preparations $(n=6)(\mathrm{f})$ in the absence and in the presence of atropine $(10 \mu \mathrm{M})$ or the presence of atropine $(10 \mu \mathrm{M})$ and $\alpha, \beta$-meATP $(10 \mu \mathrm{M})$. Representative graphs of urinary bladder contractions evoked by 20 -Hz EFS in irradiated bladder preparations and controls are displayed in the second row. $* P<0.05 ; * * P<0.01(\mathrm{~b}-\mathrm{d}) ; * P<0.05, * * P<0.01, * * * P<$ 0.001 (e and f) between basal and atropine $(10 \mu \mathrm{M})$. \#P $<0.05$, \#\#P<0.01 between atropine $(10 \mu \mathrm{M})$ and atropine $(10 \mu \mathrm{M})+\alpha, \beta$-meATP $(10 \mu \mathrm{M})$. Statistical significance was determined with the Student's $t$ test for unpaired data (a-d) or with the one-way repeated-measures ANOVA followed by the Sidak's multiple-comparisons test for paired data (e and f).

three different antibodies (see Materials and Methods). The specificity of interstitial cells could, however, not be attained with any of the assessed antibodies. c-kit staining with sab4501648 (1:500; Sigma-Aldrich) showed c-kit being expressed not only by interstitial cells, but also by smooth muscle bundles and urothelium (Fig. 5). WB (with two different antibodies) could not detect the expression of c-kit in the urinary bladder (data not shown). No expressional difference of c-kit or SCF mRNAs could be observed between irradiated bladders and controls (Fig. 5, b and c). On the protein level, SCF was also expressed similarly in irradiated bladders and controls (Fig. 5d). To further characterize the distribution and composition of interstitial cells in irradiated bladders and controls, we immunostained for platelet-derived growth factor receptor $\beta$ (PDGFr $\beta$ ), CD39L1, and CD34, which are expressed by interstitial cells of the murine urinary bladder (Yu et al., 2012). PDGFr $\beta$ was strongly expressed in the urothelium and weakly expressed in interstitial cells of the submucosa. CD34 was strongly expressed in interstitial cells in the submucosa. CD39L1 was instead only weakly expressed in interstitial cells. No expressional or distributional differences between these antigens were observed in irradiated bladders and controls (Fig. 6).

\section{Discussion}

The present study shows that bladder irradiation induces significant changes in the neuronal control of the urinary bladder. Bladder irradiation gave rise to a decrease in the bladder contraction in response to EFS, and cholinergic and purinergic agonists in vitro. EFS-evoked contractions of irradiated bladders were also less sensitive to atropine, compared with controls. Although cholinergic urinary bladder contractions in vitro were reduced in irradiated bladders, cholinergic agonists administered in vivo gave rise to similar bladder contractions in irradiated rats and controls. To note, in contrast to in vitro methacholine administration, methacholine was given at a submaximal dose in vivo, which could be one reason for more or less identical cholinergic contractions in irradiated bladders and controls. In another model for bladder pathology (cyclophosphamide-induced cystitis), we showed that bladder contractions in response to methacholine in vivo was reduced at $10 \mu \mathrm{g} / \mathrm{kg}$, but not at $5 \mu \mathrm{g} / \mathrm{kg}$, compared with controls (Aronsson et al., 2015).

Changes in the composition and function of muscarinic receptors and $\mathrm{P} 2_{\mathrm{X} 1}$ purinoceptors have been demonstrated in different models of bladder pathology such as the obstructed bladder, the diabetic bladder, the neurogenic bladder, and 
A

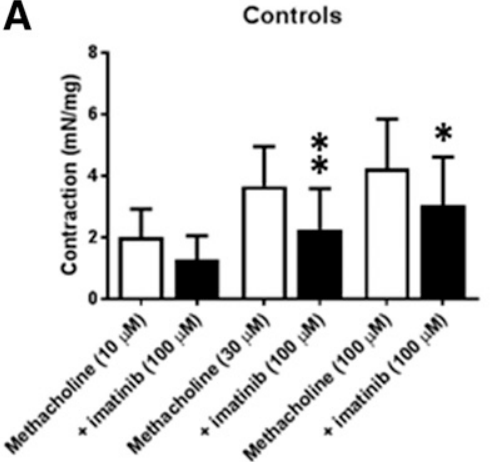

D

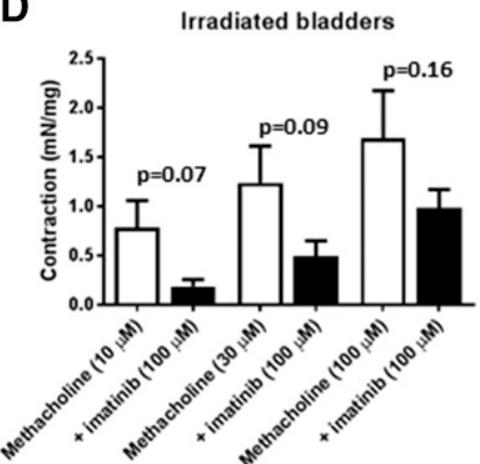

B

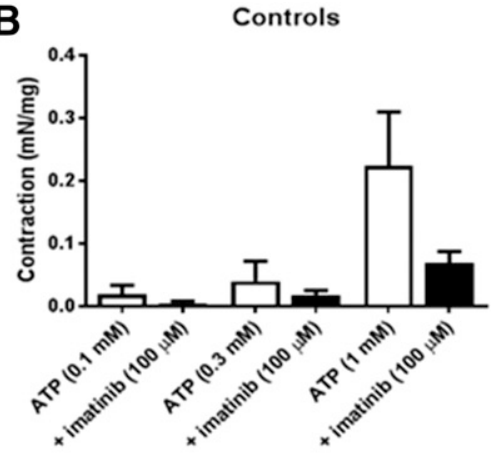

E

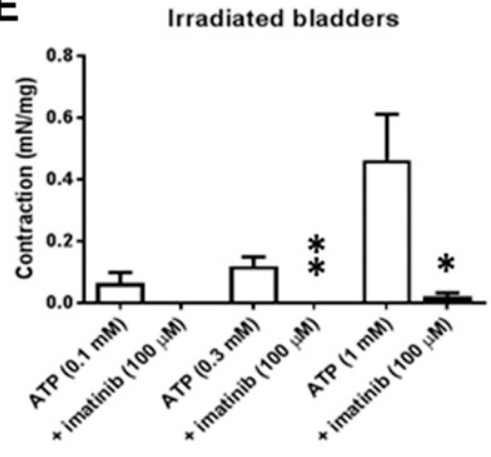

C

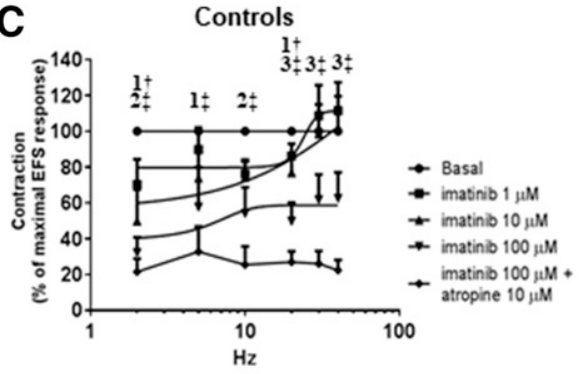

$\mathbf{F}$

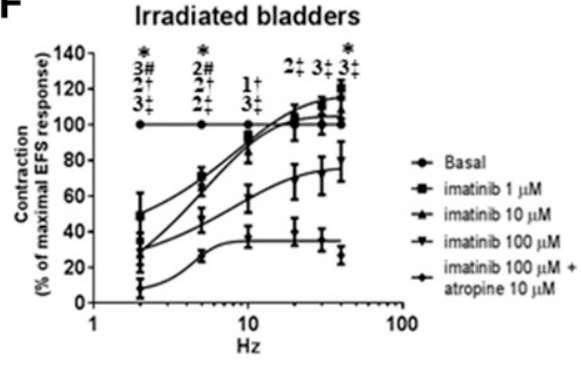

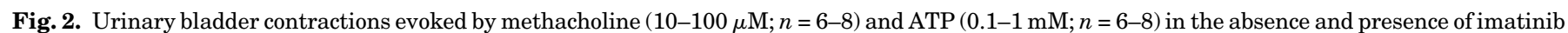

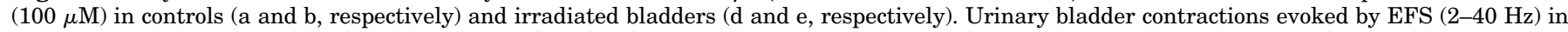

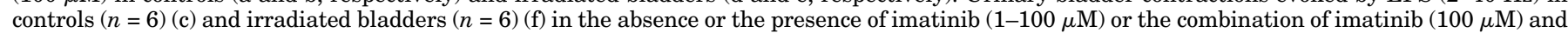

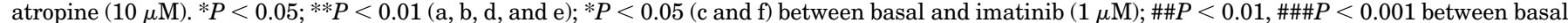

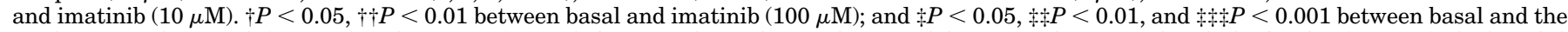

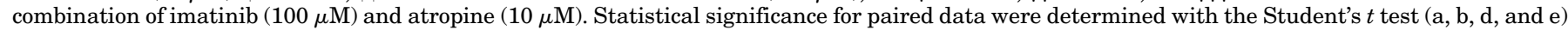
or with the one-way repeated-measures ANOVA followed by the Sidak's multiple-comparisons test (c and f).

cyclophosphamide-induced cystitis (Bayliss et al., 1999; Giglio et al., 2005; Kendig et al., 2016). Our data show that the expressions of muscarinic $\mathrm{M}_{3}$ receptors and $\mathrm{P} 2 \mathrm{X} 1$ purinoceptors were similar in irradiated bladders and controls. Therefore, we do not have evidence for a changed composition of muscarinic $\mathrm{M}_{3}$ receptors or $\mathrm{P} 2 \mathrm{X} 1$ purinoceptors contributing to the observed atropine resistance in vitro of the irradiated bladder. The observed atropine resistance in the irradiated bladder also could not be explained by changes in ChAT expression. However, acetylcholine release per se was not studied in our study. A diminished neuronal release of acetylcholine may therefore have contributed to the observed atropine resistance, since the EFS-evoked contraction was significantly reduced in response to bladder irradiation and the remaining part of the EFS-evoked contraction was only marginally affected by atropine but was almost totally antagonized by purinergic blockade. In view of KCl-evoked bladder contractions, which induce smooth muscle depolarization (Levin et al., 1991), being less affected by bladder irradiation than bladder contractions induced by exogenously administered cholinergic and purinergic agonists, we suggest that bladder irradiation predominantly affects neurons rather than inducing a general detriment of the smooth muscle function.

Although the efferent part of the neuronal control of the bladder function seemed to be depressed by bladder irradiation, the afferent part of the neuronal control seemed instead to be sensitized. Afferent EFS of the pelvic nerve gave rise to stronger contralateral bladder contractions in irradiated bladders than in controls, which leads us to suggest that bladder irradiation sensitizes the micturition reflex. The present findings are in line with those of our previous study in which we showed that bladder irradiation leads to increased bladder frequency in awake rats (Giglio et al., 2016). Interstitial cells may regulate the urinary bladder function and may be affected in bladder pathology (Kim et al., 2011; Deng et al., 2013; Canda et al., 2014). We therefore wanted to assess whether interstitial cells are involved in the changed function of the irradiated bladder. Our results show that no expressional differences could be observed in c-kit or the agonist of c-kit, SCF, either on the protein level or on the mRNA level in homogenates of irradiated bladders and controls. Conflicting data exist, however, on the presence of c-kit-positive interstitial cells in the urinary bladder. The presence of c-kit-positive interstitial cells in the rat bladder has been shown in some studies (Kim et al., 2011; Deng et al., 2012; Wu et al., 2014). Other studies have instead not been able to identify c-kit-positive interstitial cells in the rat urinary bladder (Sancho et al., 2017). Pezzone et al. (2003) reported that although interstitial cells of the murine ureteral pelvis expressed c-kit, interstitial cells of the murine urinary bladder and urethra did not (Pezzone et al., 2003). In addition 


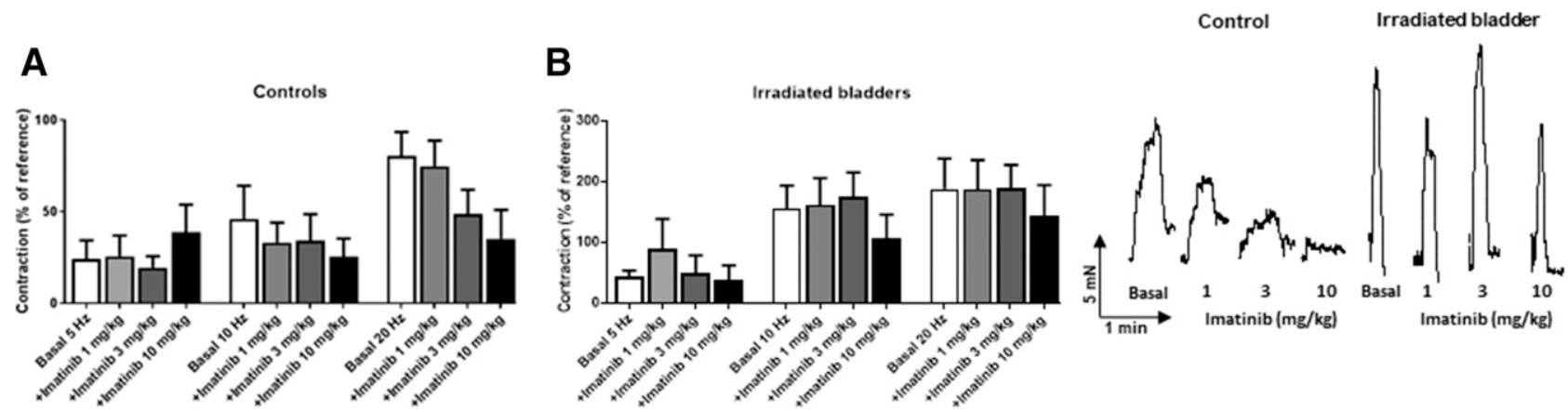

C

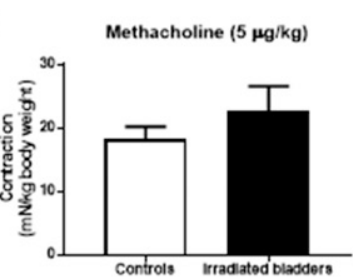

$\mathbf{F}$

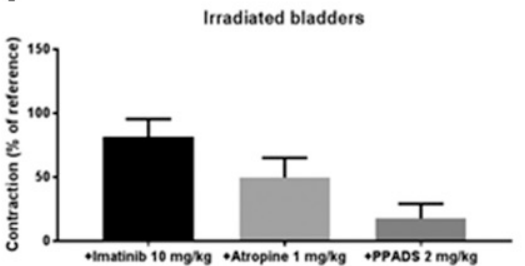

D

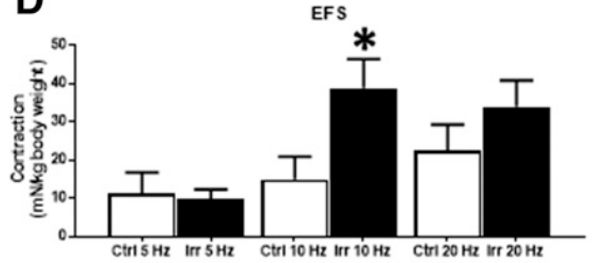

G

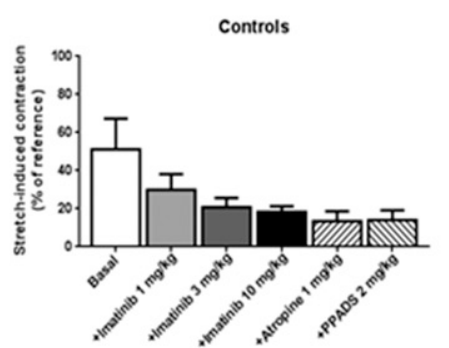

E

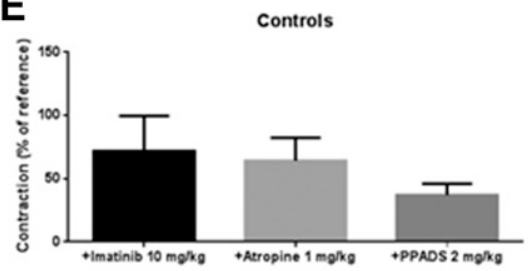

H

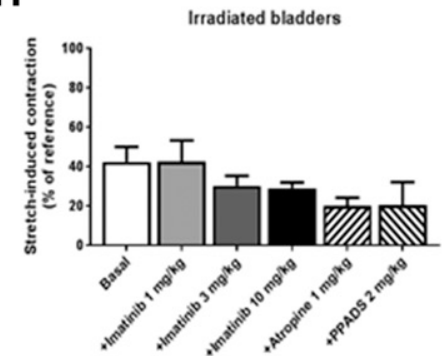

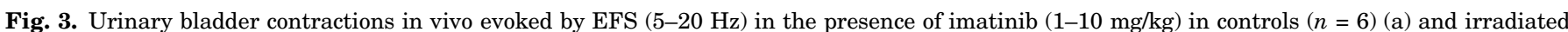

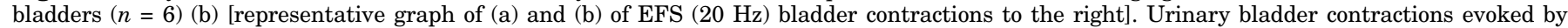

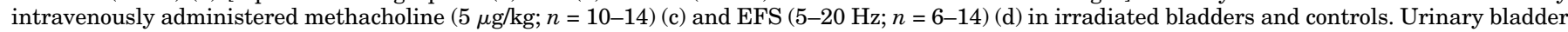

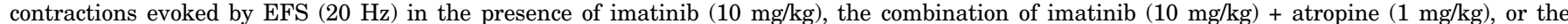

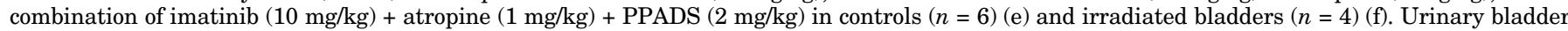

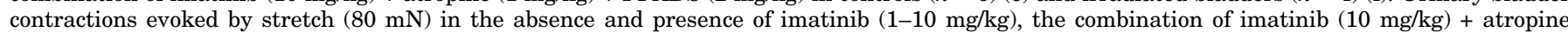

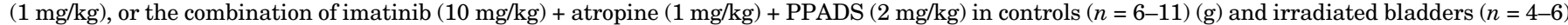

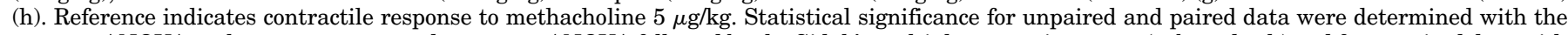

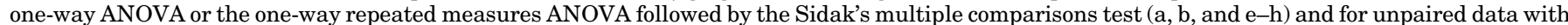
the Student's $t$ test (c and d). Ctrl, control.

to this, studies suggest that c-kit-positive interstitial cells may constitute mast cells and not pacemaker cells (Koh et al., 2018).

Of three tested antibodies, we were not able to selectively stain interstitial cells. With one of the antibodies (cat. no. sab4501648; Sigma-Aldrich), we were able to stain some interstitial cells. However, bladder smooth muscle and urothelium were also positive for c-kit. Since imatinib blocked both ATP- and methacholine-evoked contractions in vitro, c-kit may be of importance not only for interstitial cell function, but also for other functions in the urinary bladder. The interpretation of the data is also complicated by the fact that imatinib, in addition to c-kit, blocks PDGFrs (Druker et al., 1996). Our study demonstrates the expression of PDGFR $\beta$ in interstitial cells in the urinary bladder, as has been demonstrated in the murine bladder (Yu et al., 2012). Since bladder irradiation did not affect the expression of c-kit and SCF in the mRNA or protein levels, and did not affect the distribution and intensity of PDGFR $\beta, \mathrm{CD} 34$, and
CD39L1, our data suggest that interstitial cells in number and distribution are not affected 2 weeks after bladder irradiation.

Although we do not have evidence for a change in the number of interstitial cells in the urinary bladder in response to bladder irradiation, our data suggest instead that interstitial cells may modulate the neuronal control of the urinary bladder and may change in response to bladder irradiation. We presently induced stretch and afferent EFS on one part of the urinary bladder and assessed contractions on the contralateral part of the urinary bladder. Our findings show that the magnitude of contraction was linearly correlated to the frequency of EFS. Imatinib antagonized both stretch- and EFS-evoked bladder contractions in controls, but not in irradiated animals. Our findings may indicate that interstitial cells are of importance to coordinate the micturition reflex in healthy-state afferent neuronal signaling in the healthy bladder. After bladder irradiation, the regulation by interstitial cells on the micturition reflex may be changed, 

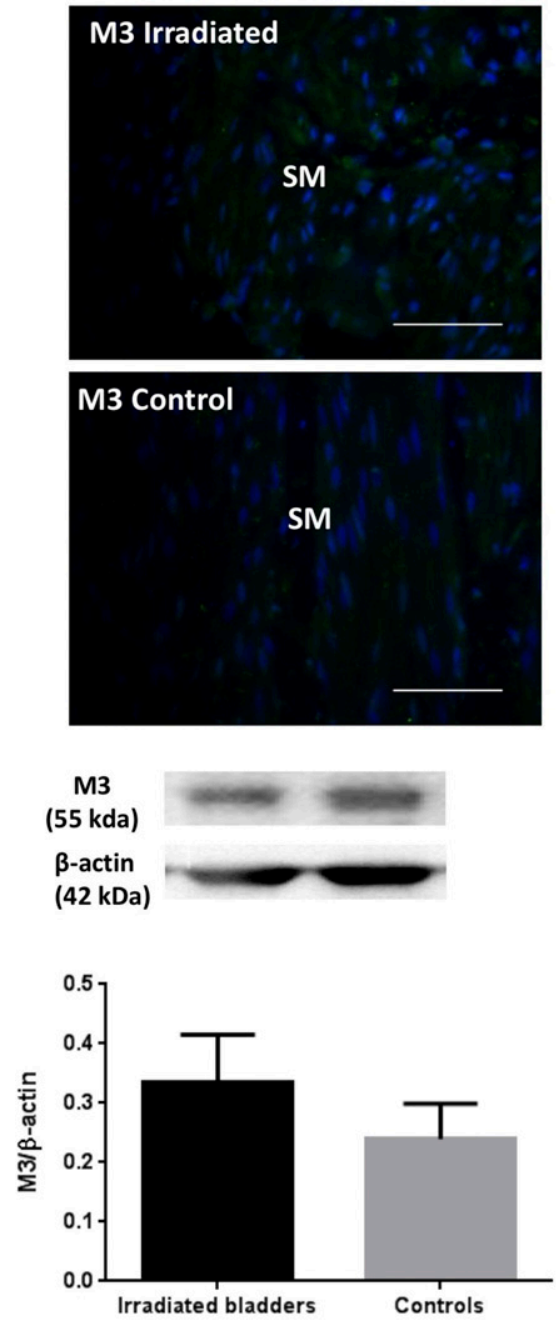
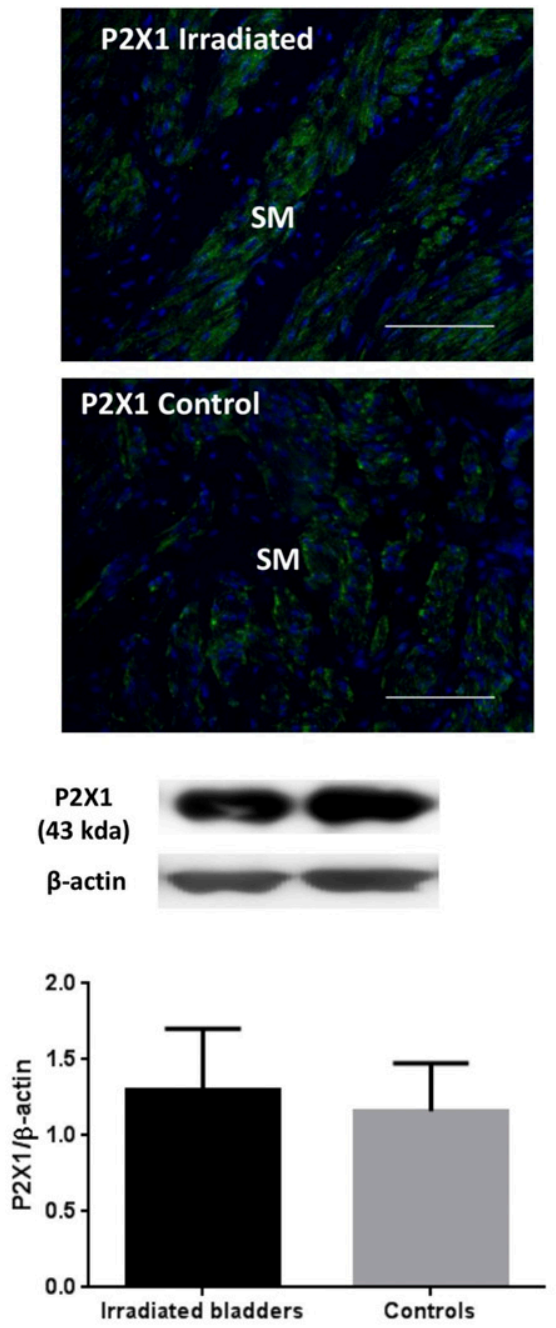
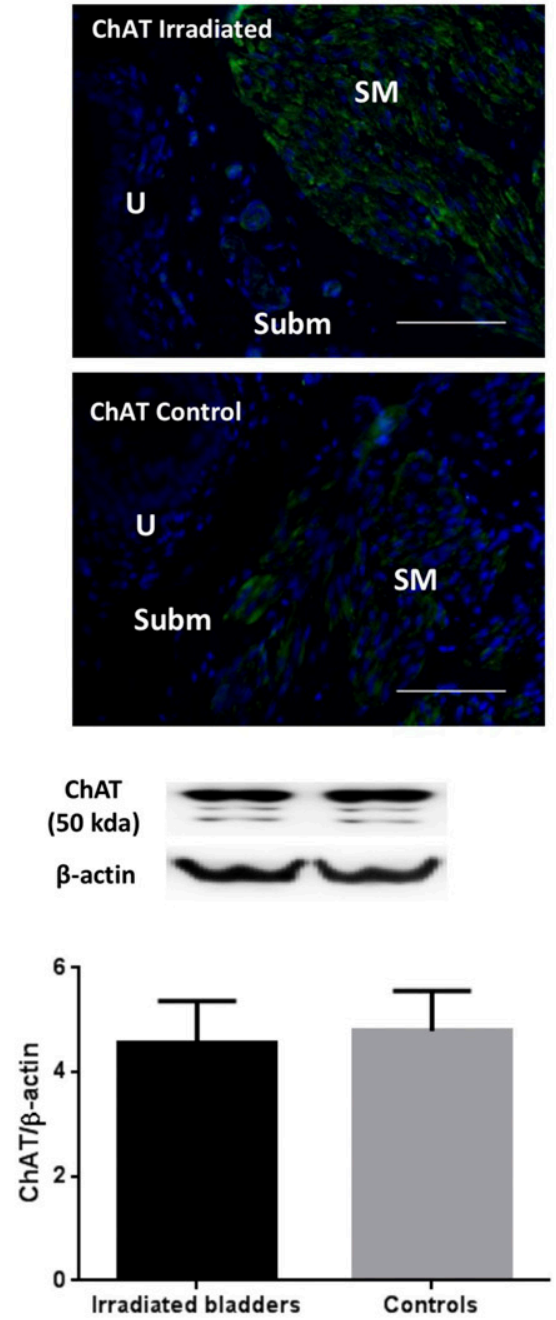

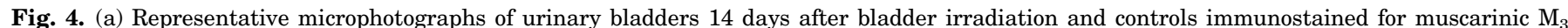

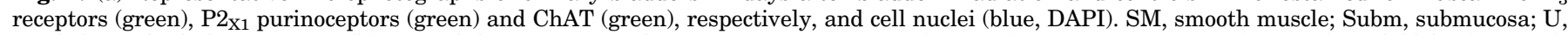

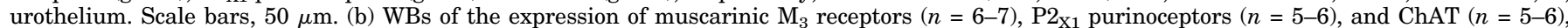

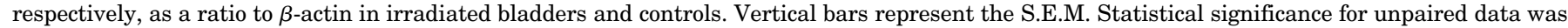
determined with the Student's $t$ test.

leading to an enhanced sensitivity from inputs from afferent neurons.

Imatinib antagonized EFS more potently at low frequencies than at high frequencies in vitro, where purinoceptors mediate the predominant part of the contraction (Aronsson et al., 2010). Imatinib also inhibited ATPevoked contractions more potently than methacholineevoked contractions in vitro. However, in vivo in control rats, imatinib dose-dependently antagonized bladder contractions induced by pelvic nerve stimulation at $20 \mathrm{~Hz}$ but not at lower frequencies. In the presence of imatinib at the highest dose $(10 \mathrm{mg} / \mathrm{kg})$, the remaining part of the EFSevoked contraction tended to be more affected by purinergic blockade than by cholinergic blockade. These findings may indicate that imatinib predominantly affects cholinergic-induced contractions in vivo. The present findings may indicate that the neuronal control of the urinary bladder may shift from a cholinergic regulation modulated by interstitial cells to a more purinergic regulation in the irradiated bladder. It is tempting to suggest that the effect of dysregulation of interstitial cells on the micturition reflex may generate some of the symptoms characterized by radiation cystitis.

Finally, it is important to mention that the expression of c-kit in interstitial cells and other cells occurs on different levels of the nervous system and the urinary bladder (Pop et al., 2013), which makes solely targeting the bladder interstitial cells difficult. The in vivo data represent the combinations of the effects of c-kit blockade on different levels, resulting in an attenuation of the afferent reflex. On the urinary bladder level, c-kit blockade had a depressing effect in both irradiated animals and controls.

In conclusion, the present findings show that bladder irradiation leads to changes on several levels in the neuronal control of the urinary bladder. Changes in the function of interstitial cells may generate some of the symptoms associated with radiation cystitis.

\section{Acknowledgments}

The authors thank the staff at the radiation facility, Department of Oncology, the Sahlgrenska University Hospital, for assistance during 
A

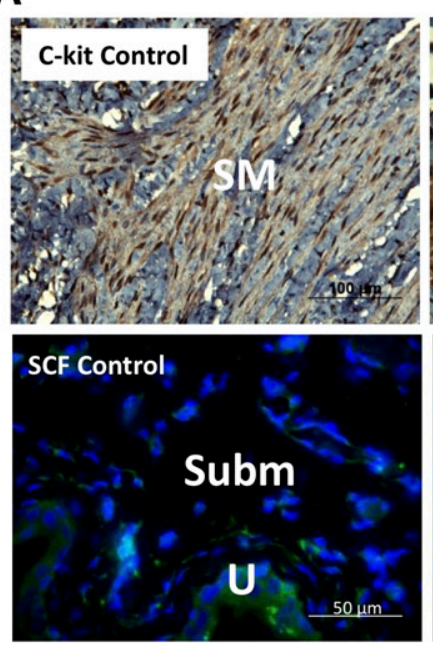

B

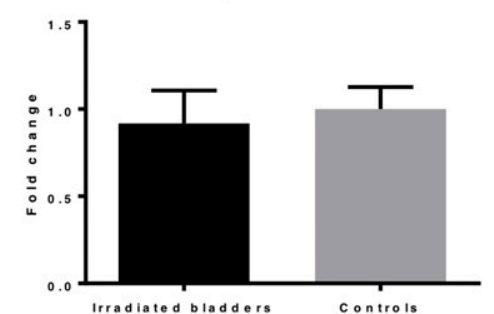

C

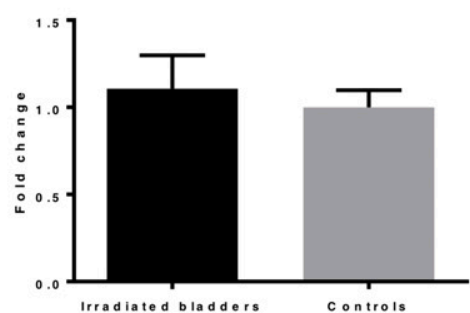

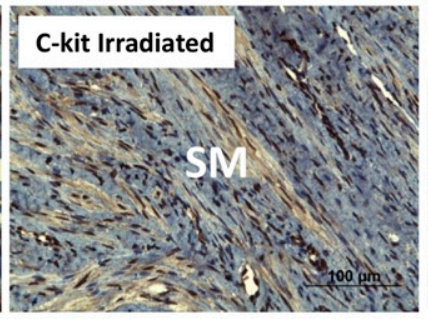
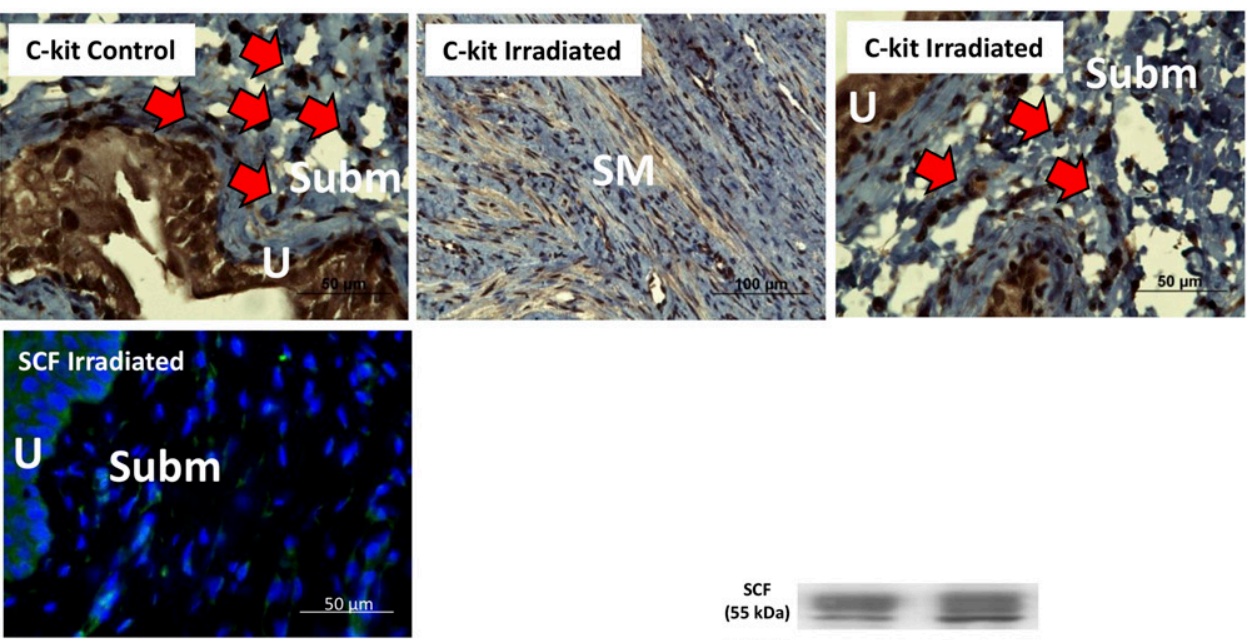

D

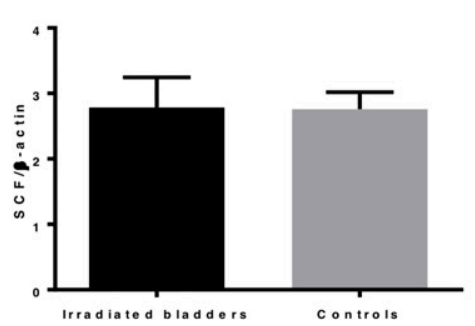

Fig. 5. (a) Representative microphotographs of urinary bladders 14 days after bladder irradiation and controls were immunostained with DAB for c-kit. Red arrows indicate interstitial cells positive for c-kit. SM, smooth muscle; Subm, submucosa; U, urothelium. qPCR analyses for c-kit (b) and SCF mRNA ( $n=6-7$ per treatment group) (c) expression; WB for SCF expression $(n=6-7$ per treatment group) (d). Vertical bars represent the S.E.M. Statistical significance for unpaired data was determined with the Student's $t$ test.

irradiation of the rats. We also thank the Histo-Center, Västra Frölunda, Sweden, for help with the preparation of urinary bladder tissues; and to Core Facilities, University of Gothenburg, for performing the qPCR analysis.

\section{Authorship Contributions}

Participated in research design: Giglio, Tobin.

Conducted experiments: Giglio, Podmolíková, Tobin.

Performed data analysis: Giglio, Podmolíková, Tobin.
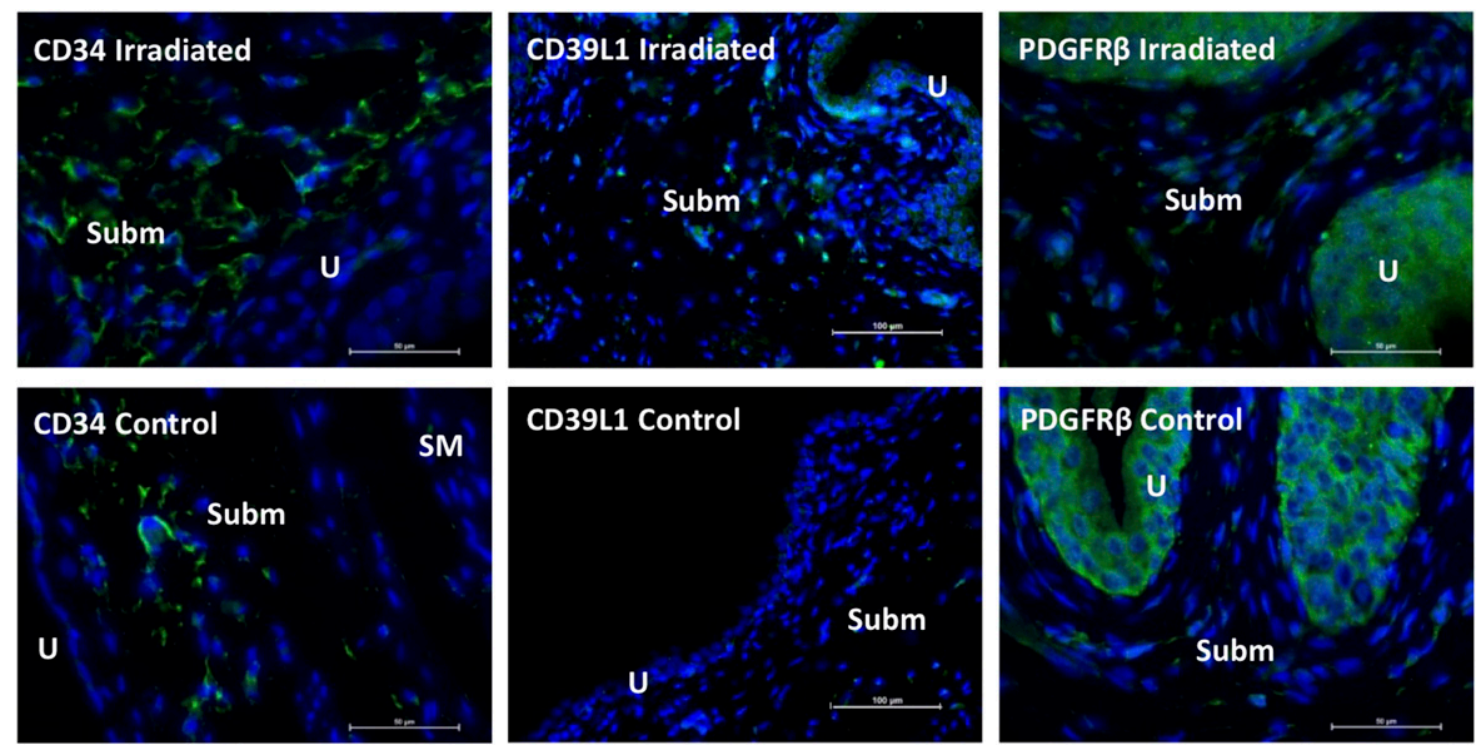

Fig. 6. Representative microphotographs of urinary bladders 14 days after bladder irradiation and controls immunostained for CD34 (green), CD39L1 (green), and PDGFr $\beta$ (green), respectively, and cell nuclei (blue, DAPI). SM, smooth muscle; Subm, submucosa; U, urothelium. 
Wrote or contributed to the writing of the manuscript: Giglio, Podmolíková, Tobin.

\section{References}

Aronsson P, Andersson M, Ericsson T, and Giglio D (2010) Assessment and characterization of purinergic contractions and relaxations in the rat urinary bladder. Basic Clin Pharmacol Toxicol 107:603-613.

Aronsson P, Carlsson T, Winder M, and Tobin G (2014) A novel in situ urinary bladder model for studying afferent and efferent mechanisms in the micturition reflex in the rat. Neurourol Urodyn 33:550-557.

Aronsson P, Carlsson T, Winder M, and Tobin G (2015) Cyclophosphamide-induced alterations of the micturition reflex in a novel in situ urinary bladder model in the anesthetized rat. Neurourol Urodyn 34:375-380.

Bayliss M, Wu C, Newgreen D, Mundy AR, and Fry CH (1999) A quantitative study of atropine-resistant contractile responses in human detrusor smooth muscle, from stable, unstable and obstructed bladders. J Urol 162:1833-1839.

Brading AF and McCloskey KD (2005) Mechanisms of disease: specialized interstitial cells of the urinary tract-an assessment of current knowledge. Nat Clin Pract Urol 2:546-554

Canda AE, Dogan H, Kandemir O, Atmaca AF, Akbulut Z, and Balbay MD (2014) Does diabetes affect the distribution and number of interstitial cells and neuronal tissue in the ureter, bladder, prostate, and urethra of humans? Cent European $J$ Urol 67:366-374.

Deng J, He P, Zhong X, Wang Q, Li L, and Song B (2012) Identification of T-type calcium channels in the interstitial cells of Cajal in rat bladder. Urology 80:1389. e1-7.

Deng J, Zhang Y, Wang L, Zhao J, Song B, and Li L (2013) The effects of Glivec on the urinary bladder excitation of rats with suprasacral or sacral spinal cord transection. J Surg Res 183:598-605.

Druker BJ, Tamura S, Buchdunger E, Ohno S, Segal GM, Fanning S, Zimmermann $J$, and Lydon NB (1996) Effects of a selective inhibitor of the Abl tyrosine kinase on the growth of Bcr-Abl positive cells. Nat Med 2:561-566.

Gevaert T, Vanstreels E, Daelemans D, Franken J, Van Der Aa F, Roskams T, and De Ridder D (2014) Identification of different phenotypes of interstitial cells in the upper and deep lamina propria of the human bladder dome. J Urol 192 $1555-1563$.

Giglio D, Ryberg AT, To K, Delbro DS, and Tobin G (2005) Altered muscarinic receptor subtype expression and functional responses in cyclophosphamide induced cystitis in rats. Auton Neurosci 122:9-20.

Giglio D, Wasén C, Mölne J, Suchy D, Swanpalmer J, Jabonero Valbuena J, Tobin G, and Ny L (2016) Downregulation of toll-like receptor 4 and IL-6 following irradiation of the rat urinary bladder. Clin Exp Pharmacol Physiol 43:698-705.

Kendig DM, Ets HK, and Moreland RS (2016) Effect of type II diabetes on male rat bladder contractility. Am J Physiol Renal Physiol 310:F909-F922.

Kim SO, Oh BS, Chang IY, Song SH, Ahn K, Hwang EC, Oh KJ, Kwon D, and Park K (2011) Distribution of interstitial cells of Cajal and expression of nitric oxide synthase after experimental bladder outlet obstruction in a rat model of bladder overactivity. Neurourol Urodyn 30:1639-1645.
Koh SD, Lee H, Ward SM, and Sanders KM (2018) The mystery of the interstitial cells in the urinary bladder. Annu Rev Pharmacol Toxicol 58:603-623.

Kubota Y, Biers SM, Kohri K, and Brading AF (2006) Effects of imatinib mesylate (Glivec) as a c-kit tyrosine kinase inhibitor in the guinea-pig urinary bladder. Neurourol Urodyn 25:205-210.

Levin RM, Hypolite J, Longhurst PA, and Wein AJ (1991) Comparison of the contractile and metabolic effects of muscarinic stimulation with those of $\mathrm{KCl}$. Pharmacology 42:142-150.

McCloskey KD and Gurney AM (2002) Kit positive cells in the guinea pig bladder. $J$ Urol 168:832-836.

Michailov MC, Neu E, Tempel K, Hölzl H, and Breiter N (1991) Influence of $\mathrm{X}$-irradiation on the motor activity of rat urinary bladder in vitro and in vivo. Strahlenther Onkol 167:311-318.

Min Y, He P, Wang Q, Jin X, Song B, and Li L (2011) The effects of the c-kit blocker glivec on the contractile response of urinary bladder. J Surg Res 171:e193-e199.

Oscarsson N, Ny L, Mölne J, Lind F, Ricksten SE, Seeman-Lodding H, and Giglio D (2017) Hyperbaric oxygen treatment reverses radiation induced pro-fibrotic and oxidative stress responses in a rat model. Free Radic Biol Med 103:248-255.

Pezzone MA, Watkins SC, Alber SM, King WE, de Groat WC, Chancellor MB, and Fraser MO (2003) Identification of c-kit-positive cells in the mouse ureter: the interstitial cells of Cajal of the urinary tract. Am J Physiol Renal Physiol 284: F925-F929.

Pop E, Mărdărescu M, Lazăr M, Rusu MC, and Ion DA (2013) c-Kit expression in somatosensory nuclei of lower medulla oblongata. Rom J Morphol Embryol 54 (3 Suppl):721-724.

Sancho M, Triguero D, Lafuente-Sanchis A, and Garcia-Pascual A (2017) Proliferation of interstitial cells in the cyclophosphamide-induced cystitis and the preventive effect of imatinib. BioMed Res Int 2017:3457093.

Soler R, Vianello A, Füllhase C, Wang Z, Atala A, Soker S, Yoo JJ, and Koudywilliam J (2011) Vascular therapy for radiation cystitis. Neurourol Urodyn 30:428-434.

Vale JA, Liu K, Whitfield HN, and Trott KR (1994) Post-irradiation bladder dysfunction: muscle strip findings. Urol Res 22:51-55.

van der AA F, Roskams T, Blyweert W, Ost D, Bogaert G, and De Ridder D (2004) Identification of kit positive cells in the human urinary tract. $J$ Urol 171: 2492-2496.

Wu Y, Shi C, Deng J, Zhang X, Song B, and Li L (2014) Expression and function of muscarinic subtype receptors in bladder interstitial cells of cajal in rats. Urol $J$ 11: $1642-1647$.

Yu W, Zeidel ML, and Hill WG (2012) Cellular expression profile for interstitial cells of cajal in bladder - a cell often misidentified as myocyte or myofibroblast. PLoS One 7:e48897.

Zwaans BM, Chancellor MB, and Lamb LE (2016) Modeling and treatment of radiation cystitis. Urology 88:14-21.

Address correspondence to: Daniel Giglio, Department of Pharmacology, University of Gothenburg, BOX 431, SE-405 30 Gothenburg, Sweden. E-mail: daniel.giglio@pharm.gu.se 